\title{
Hydrodynamic and Hydromagnetic Stability of Viscous-Viscoelastic Superposed Fluids in Presence of Suspended Particles
}

\author{
Pardeep Kumar and Mahinder Singh \\ Received 11 December 2006; Accepted 2 May 2007 \\ Recommended by Gernot Krammer
}

The Rayleigh-Taylor instability of a Newtonian viscous fluid overlying an Oldroydian viscoelastic fluid containing suspended particles is considered. As in both Newtonian viscous-viscous fluids, the system is stable in the potentially stable case and unstable in the potentially unstable case, this holds for the present problem also. The effect of a variable horizontal magnetic field is also considered. The presence of magnetic field stabilizes a certain wavenumber band, whereas the system is unstable for all wavenumbers in the absence of the magnetic field for the potentially unstable arrangement.

Copyright $\odot 2007$ P. Kumar and M. Singh. This is an open access article distributed under the Creative Commons Attribution License, which permits unrestricted use, distribution, and reproduction in any medium, provided the original work is properly cited.

\section{INTRODUCTION}

A comprehensive account of the instability of the plane interface between two Newtonian fluids, under varying assumptions of hydrodynamics and hydromagnetics, has been given by Chandrasekhar [1]. The nature of the instability and some other factors may have different effects on viscoelastic fluids as compared to Newtonian fluids. For example, Bhatia and Steiner [2] have studied the problem of thermal instability of Maxwellian viscoelastic fluid in the presence of rotation and found that the rotation has a destabilizing effect, in contrast to the stabilizing effect on Newtonian fluid. Bhatia and Steiner [3] have also considered the thermal instability of a Maxwell fluid in hydromagnetics and have found that magnetic field has a stabilizing effect on viscoelastic fluid as in the case of Newtonian fluid. Sharma [4] studied the thermal instability of a layer of Oldroyd fluid acted on by a uniform rotation and found that the rotation has a destabilizing as well as a stabilizing effect under certain conditions, in contrast to a Maxwell (viscoelastic) fluid, where it has destabilizing effect (Bhatia and Steiner [2]).

In geophysical situations, more often than not, the fluid is not pure but may instead be permeated with suspended (or dust) particles. The effect of suspended particles on the stability of superposed fluids might be of industrial and chemical engineering importance. Further, motivation for this study is the fact that knowledge concerning fluid-particle mixtures is not commensurate with their industrial and scientific importance. Scanlon and Segel [5] have considered the effect of suspended particles on the onset of Bénard convection and found that the critical Rayleigh number was reduced solely because the heat capacity of the pure gas was supplemented by that of the particles. Sharma et al. [6] have studied the effect of suspended particles on the onset of Bénard convection in hydromagnetics. The effect of suspended particles was found to destabilize the layer whereas the effect of a magnetic field was stabilizing. Palaniswamy and Purushotham [7] have studied the stability of shear flow of stratified fluids with fine dust and found the effect of fine dust to increase the region of instability. Recently, spacecraft observations have confirmed that the dust particles play an important role in the dynamics of atmosphere as well as in the diurnal and surface variations in the temperature of the Martian weather. It is, therefore, of interest to study the presence of suspended particles in astrophysical situations.

Attention has recently been drawn by calculations of the rheological behavior of dilute suspensions and emulsions (Frohlish and Sack [8] and Oldroyd [9]) to the idealized incompressible viscoelastic liquids whose behavior at small variable shear stresses is characterized by three parameters, that is, a coefficient of viscosity $\mu$, a relaxation time $\lambda$, and a retardation time $\lambda_{0}(<\lambda)$. Oldroyd [10] proposed a theoretical model, for a class of viscoelastic fluids. An experimental demonstration by Toms and Strawbridge [11] reveals that 
a dilute solution of methyl methacrylate in $n$-butyl acetate agrees well with the theoretical model of the Oldroyd fluid. R. C. Sharma and K. C. Sharma [12] studied the stability of the plane interface separating two Oldroydian viscoelastic superposed fluids of uniform densities.

Usually the magnetic field has a stabilizing effect on the instability. However, Kent [13] has studied the effect of a horizontal magnetic field on the stability of parallel flows, which varies in the vertical direction and has shown that system is unstable under certain conditions, while in absence of magnetic field the system is known to be stable. In the interior of the Earth, the magnetic field may be variable and may altogether alter the nature of the instability. The Rayleigh-Taylor instability of a plane interface between viscous and viscoelastic fluid through a porous medium has been investigated by Sharma and Kumar [14]. They concluded that the top heavy arrangement is unstable in the absence or in the presence of a magnetic field, this result being same as for a Newtonian fluid. Agrawal and Goel [15] have studied the instability of viscoelastic fluid in a porous medium and their analysis reveals a stabilizing character of viscosity and viscoelasticity and a destabilizing character of medium permeability and the shear velocity. Bhatia and Mathur [16] have studied the instability of viscoelastic fluids in a vertical magnetic field through porous medium and it is found that the magnetic field stabilizes the unstable configuration for wavenumber band $k>k^{*}$ in which the system is unstable in the absence of the magnetic field. It is also found that the viscosity, viscoelasticity, and medium porosity have stabilizing influence while elasticity and medium permeability have destabilizing influence. Kumar et al. [17] have considered the instability of the plane interface between two Oldroydian viscoelastic superposed fluids in the presence of uniform rotation and variable magnetic field in porous medium.

The instability of a plane interface between viscous (Newtonian) and viscoelastic (Oldroydian) fluids containing suspended particles may be of interest in geophysics, chemical technology, and biomechanics and is therefore studied in the present paper. The effect of a variable horizontal magnetic field, bearing relevancy in geophysics, is also considered.

\section{FORMULATION OF THE PROBLEM AND PERTURBATION EQUATIONS}

Let $T_{i j}, \tau_{i j}, e_{i j}, \mu, \lambda, \lambda_{0}(<\lambda), p, \delta_{i j}, v_{i}, x_{i}$, and $d / d t$ denote respectively, the total stress tensor, the shear stress tensor, the rate-of-strain tensor, the viscosity, the stress relaxation time, the strain retardation time, the isotropic pressure, the Kronecker delta, the velocity vector, the position vector, and the mobile operator. Then the Oldroydian viscoelastic fluid is described by the constitutive relations

$$
\begin{gathered}
T_{i j}=-p \delta_{i j}+\tau_{i j} \\
\left(1+\lambda \frac{d}{d t}\right) \tau_{i j}=2 \mu\left(1+\lambda_{0} \frac{d}{d t}\right) e_{i j} \\
e_{i j}=\frac{1}{2}\left(\frac{\partial v_{i}}{\partial x_{j}}+\frac{\partial v_{j}}{\partial x_{i}}\right)
\end{gathered}
$$

Relations of the type (1) were proposed and studied by Oldroyd [10]. Oldroyd showed that many rheological equations of general validity reduce to (1) when linearized. $\lambda_{0}=0$ yields the Maxwellian fluid, whereas $\lambda=\lambda_{0}=0$ gives the Newtonian viscous fluid.

Consider a static state in which an incompressible Oldroydian viscoelastic fluid containing suspended particles is arranged in horizontal strata and the pressure $p$ and the density $\rho$ are functions of the vertical coordinate $z$ only. The character of the equilibrium of this initial static state is determined, as usual, by supposing that the system is slightly disturbed and then following its further evolution.

Let $\vec{v}(u, v, w), \rho$ and $\mathrm{p}$ denote, respectively, the velocity of pure fluid, the density and the pressure; $\vec{u}(\bar{x}, t)$ and $\mathrm{N}(\bar{x}, t)$ denote the velocity and the number density of the suspended particles, respectively. $K=6 \pi \rho v \eta$, where $\eta$ is the particle radius, is the Stokes' drag coefficient, $\vec{u}=(\ell, r, s), \bar{x}=(x, y, z)$ and $\lambda^{\prime}=(0,0,1)$. The system is acted on by a gravity field $\vec{g}(0,0,-g)$. The theory assumes the relative motion between fluid and particles to be slow (as the Stokes drag formula applies only to low Reynolds numbers), therefore, the equations of motion and continuity for the Oldroydian viscoelastic fluid containing suspended particles are

$$
\begin{gathered}
\rho\left(1+\lambda \frac{\partial}{\partial t}\right)\left[\frac{\partial \vec{v}}{\partial t}+(\vec{v} \cdot \nabla) \vec{v}\right] \\
=\left(1+\lambda \frac{\partial}{\partial t}\right)\left[-\nabla p+\rho \vec{g} \lambda^{\prime}+K N(\vec{u}-\vec{v})\right] \\
+\mu\left(1+\lambda_{0} \frac{\partial}{\partial t}\right) \nabla^{2} \vec{v} \\
\nabla \cdot \vec{v}=0 .
\end{gathered}
$$

Since the density of a fluid particle remains unchanged as we follow it with its motion, we have

$$
\frac{\partial \rho}{\partial t}+(\vec{v} \cdot \nabla) \rho=0 .
$$

In the equations of motion (2), by assuming a uniform spherical particle shape and small relative velocities between the fluid and particles, the presence of particles adds on extra force term proportional to the velocity difference between the particles and the fluid. Since the force exerted by the fluid on the particles is equal and opposite to that exerted by the particles on the fluid, there must be an extra force term, equal in magnitude but opposite in sign, in the equations of motion of the particles. The distances between particles are assumed quite large compared with their diameter so that interparticle reactions are ignored. The effects of pressure and gravity on the suspended particles are negligibly small and therefore ignored. If $m N$ is the mass of particles per unit volume, then the equations of motion and continuity for the particles, under the above assumptions, are

$$
\begin{gathered}
m N\left[\frac{\partial \vec{u}}{\partial t}+(\vec{u} \cdot \nabla) \vec{u}\right]=K N(\vec{v}-\vec{u}), \\
\frac{\partial N}{\partial t}+\nabla \cdot(N \vec{u})=0 .
\end{gathered}
$$


Let $\vec{v}(u, v, w), \delta \rho, \delta p$ and $\vec{u}(l, r, s)$ denote, respectively, the perturbations in fluid velocity $(0,0,0)$, fluid density $\rho$, fluid pressure $p$, and particle velocity $(0,0,0)$. Then the linearized perturbation equations of the fluid-particle layer are

$$
\begin{aligned}
& \rho\left(1+\lambda \frac{\partial}{\partial t}\right) \frac{\partial \vec{v}}{\partial t}=\left(1+\lambda \frac{\partial}{\partial t}\right)[-\nabla \delta p+\vec{g} \delta \rho+K N(\vec{u}-\vec{v})] \\
&+ \mu\left(1+\lambda_{0} \frac{\partial}{\partial t}\right) \nabla^{2} \vec{v} \\
& \nabla \cdot \vec{v}=0 \\
& \frac{\partial}{\partial t} \delta \rho=-w(D \rho) \\
&\left(\frac{m}{K} \frac{\partial}{\partial t}+1\right) \vec{u}=\vec{v} \\
& \frac{\partial M}{\partial t}+\nabla \cdot \vec{u}=0
\end{aligned}
$$

where $M=N / N_{0}$ and $N_{0}, N$ stand for initial uniform number density and perturbation in number density, respectively, and $D=d / d z$.

Analyzing the disturbances into normal modes, we seek solutions whose dependence on $x, y$, and $t$ is given by

$$
\exp \left(i k_{x} x+i k_{y} y+n t\right)
$$

where $k_{x}, k_{y}$ are horizontal wavenumbers, $k^{2}=k_{x}^{2}+k_{y}^{2}$, and $n$ is a complex constant.

For perturbations of the form (12), (7)-(10) after eliminating $\vec{u}$ give

$$
\begin{aligned}
& {\left[\rho+\frac{m N}{\tau n+1}\right](1+\lambda n) n u} \\
& \quad=(1+\lambda n)\left(-i k_{x} \delta p\right)+\left(1+\lambda_{0} n\right) \mu\left(D^{2}-k^{2}\right) u
\end{aligned}
$$

$i k_{x} u+i k_{y} v+D w=0$,

$n \delta \rho=-w(D \rho)$,

where $\tau=m / K$.

Eliminating $\delta p$ between (13)-(15) and using (16) and (17), we obtain

$$
\begin{aligned}
& n(1+\lambda n)\left[D(\rho D w)-k^{2} \rho w\right] \\
&+ \frac{n(1+\lambda n)}{\tau n+1}\left[D(m N D w)-k^{2} m N w\right] \\
&-\left(1+\lambda_{0} n\right)\left(D^{2}-k^{2}\right)\left[D(\mu D w)-k^{2} \mu w\right] \\
&+\frac{(1+\lambda n) g k^{2}(D \rho)}{n}=0 .
\end{aligned}
$$

\section{TWO UNIFORM VISCOUS AND VISCOELASTIC (OLDROYDIAN) FLUIDS SEPARATED BY A HORIZONTAL BOUNDARY}

Consider the case of two uniform fluids of densities, viscosities, suspended particles number densities; $\rho_{2}, \mu_{2}, N_{2}$ (upper Newtonian fluid), and $\rho_{1}, \mu_{1}, N_{1}$ (lower Oldroydian viscoelastic fluid) separated by a horizontal boundary at $z=0$. Then, in each region of constant $\rho$, constant $\mu$, and constant $m N$, (18) reduces to

$$
\left(D^{2}-k^{2}\right)\left(D^{2}-q^{2}\right) w=0,
$$

here

$$
q^{2}=k^{2}+\frac{n(1+\lambda n) \rho}{\left(1+\lambda_{0} n\right) \mu}+\frac{n(1+\lambda n) m N}{(\tau n+1)\left(1+\lambda_{0} n\right) \mu} .
$$

Since $w$ must vanish both when $z \rightarrow+\infty$ (in the upper fluid) and $z \rightarrow-\infty$ (in the lower fluid), the general solution of (19) can be written as

$$
\begin{array}{ll}
w_{1}=A_{1} e^{+k z}+B_{1} e^{+q_{1} z} & (z<0), \\
w_{2}=A_{2} e^{-k z}+B_{2} e^{-q_{2} z} & (z>0),
\end{array}
$$

where $A_{1}, B_{1}, A_{2}, B_{2}$ are constants of integration,

$$
\begin{gathered}
q_{1}=\left[k^{2}+\frac{n(1+\lambda n) \rho_{1}}{\left(1+\lambda_{0} n\right) \mu_{1}}+\frac{n(1+\lambda n) m N}{(1+\tau n)\left(1+\lambda_{0} n\right) \mu_{1}}\right]^{1 / 2}, \\
q_{2}=\left[k^{2}+\frac{n \rho_{2}}{\mu_{2}}+\frac{n m N}{(1+\tau n) \mu_{2}}\right]^{1 / 2} .
\end{gathered}
$$

In writing (22), it is assumed that $q_{1}$ and $q_{2}$ are so defined that their real parts are positive.

\section{BOUNDARY CONDITIONS}

The solutions (21) must satisfy certain boundary conditions. Assuming that the second horizontal velocity component $v$ vanishes and that the surface tension at the interface is neglected, the boundary conditions to be satisfied at the interface $z=0$ are

$$
\begin{gathered}
w, \\
D w \\
\mu\left(D^{2}+k^{2}\right) w .
\end{gathered}
$$

They must be continuous across the interface between two fluids. Physically (23) and (24) result from the continuity of velocity and (25) from the continuity of shear stress. Integrating (18) across the interface at $z=0$, we obtain another condition which results from the balance of the normal stresses (together with the kinematic free surface condition) as

$$
\begin{aligned}
& n\left[\rho_{2} D w_{2}-\rho_{1}(1+\lambda n) D w_{1}\right]_{z=0} \\
& \quad+\frac{n m}{\tau n+1}\left[N_{2} D w_{2}-(1+\lambda n) N_{1} D w_{1}\right]_{z=0} \\
& \quad-\left[\mu_{2}\left(D^{2}-k^{2}\right) D w_{2}-\mu_{1}\left(1+\lambda_{0} n\right)\left(D^{2}-k^{2}\right) D w_{1}\right]_{z=0} \\
& \quad+\frac{g k^{2}}{n}\left[\rho_{2}-(1+\lambda n) \rho_{1}\right] w_{0}=0
\end{aligned}
$$


Remember that upper fluid is Newtonian and lower Oldroydian viscoelastic. $w_{0}$ is the common value of $w$ at $z=0$.

\section{DISPERSION RELATION AND DISCUSSION}

Applying the boundary conditions (23)-(26) to the solutions given in (21), we obtain

$$
\begin{gathered}
A_{1}+B_{1}=A_{2}+B_{2} \quad\left(=w_{0}\right), \\
k A_{1}+q_{1} B_{1}=-k A_{2}-q_{2} B_{2} \quad\left(=D w_{0}\right), \\
\mu_{1}\left[2 k^{2} A_{1}+\left(q_{1}^{2}+k^{2}\right) B_{1}\right] \\
=\mu_{2}\left[2 k^{2} A_{2}+\left(q_{2}^{2}+k^{2}\right) B_{2}\right] \quad\left(=\mu\left(D^{2}+k^{2}\right)\right) w_{0}, \\
{\left[\rho_{1}+\frac{m N_{1}}{\tau n+1}-\frac{R}{2}\right] A_{1}} \\
+\left[\frac{\rho_{1} q_{1}}{k}+\frac{m N_{1} q_{1}}{k(\tau n+1)}-\frac{\left(1+\lambda_{0} n\right) \mu_{1} q_{1}\left(q_{1}^{2}-k^{2}\right)}{k n(1+\lambda n)}-\frac{R}{2}\right] B_{1} \\
+\frac{1}{(1+\lambda n)}\left[\rho_{2}+\frac{m N_{2}}{\tau n+1}-\frac{R}{2}\right] A_{2} \\
+\frac{1}{(1+\lambda n)}\left[\frac{\rho_{2} q_{2}}{k}+\frac{m N_{2} q_{2}}{k(\tau n+1)}-\frac{\mu_{2} q_{2}\left(q_{2}^{2}-k^{2}\right)}{n k}-\frac{R}{2}\right] B_{2} \\
=0,
\end{gathered}
$$

where $R=\left(g k / n^{2}\right)\left(\rho_{2}-\rho_{1}\right)$.

For getting nontrivial solutions $\left(A_{1}, B_{1}, A_{2}, B_{2}\right)$, the determinant of the linear system of (27) must clearly vanish. The determinant can be reduce by subtracting its first column from the second, the third column from the fourth and adding the first column to the third. By this procedure, we obtain

$$
\left|\begin{array}{ccc}
1 & 2 k & 1 \\
\mu_{1}\left(q_{1}+k\right) & -2 k^{2}\left(\mu_{2}-\mu_{1}\right) & -\mu_{2}\left(q_{2}+k\right) \\
{\left[\frac{\rho_{1}}{k}+\frac{m N_{1}}{k(\tau n+1)}\right.} & {\left[\frac{1}{(1+\lambda n)}\right.} & {\left[\frac{\rho_{2}}{k(1+\lambda n)}\right.} \\
\left.-\frac{\left(1+\lambda_{0} n\right) \mu_{1} q_{1}\left(q_{1}+k\right)}{k n(1+\lambda n)}\right] & \times\left\{\rho_{2}+\frac{m N_{2}}{\tau n+1}-\frac{R}{2}\right\} & +\frac{m N_{2}}{k(\tau n+1)(1+\lambda n)} \\
& \left.+\left\{\rho_{1}+\frac{m N_{1}}{\tau n+1}-\frac{R}{2}\right\}\right] & \left.-\frac{\mu_{2} q_{2}\left(q_{2}+k\right)}{n k(1+\lambda n)}\right]
\end{array}\right|
$$

$=0$.

The dispersion relation (28) is quite complicated as the values of $q_{1}$ and $q_{2}$ involve square roots. For mathematical simplicity, we make the assumptions that the kinematic viscosities of both fluids to be equal, that is, $v_{1}=v_{2}=v$ (Chandrasekhar; [1, page 443]), $m N / \rho=m N_{1} / \rho_{1}=m N_{2} / \rho_{2}$ and that the fluids are highly viscous, as these simplifying assumptions do not obscure any of the essential features of the problem, under the above assumptions, we have

$$
q=k\left[1+\frac{m(1+\lambda n)}{2 \nu k^{2}\left(1+\lambda_{0} n\right)}+\frac{n(1+\lambda n) m N}{2 \rho \nu k^{2}(1+\tau n)\left(1+\lambda_{0} n\right)}\right]
$$

so that

$$
\begin{gathered}
q_{1}=k\left[1+\frac{n(1+\lambda n)}{2 v k^{2}\left(1+\lambda_{0} n\right)}+\frac{n(1+\lambda n) m N}{2 \rho_{1} v k^{2}(1+\tau n)\left(1+\lambda_{0} n\right)}\right] \\
q_{2}=k\left[1+\frac{n}{2 v k^{2}}+\frac{n m N}{2 \rho_{2} v k^{2}(1+\tau n)}\right]
\end{gathered}
$$

remembering that upper fluid is Newtonian and lower Oldroydian viscoelastic.

Substituting the values of $q_{1}+k$ and $q_{2}+k$ from the expressions (30) in the determinant (28) and simplifying it, after a little algebra, we obtain

$$
A_{9} n^{9}+A_{8} n^{8}+\cdots+A_{2} n^{2}+A_{1} n+A_{0}=0,
$$

where

$$
\begin{gathered}
A_{9}=\lambda^{2} \tau^{3} \rho_{1} \rho_{2}, \\
A_{0}=-8 k^{6} v^{2} g\left(\rho_{2}-\rho_{1}\right)\left(\rho_{1}+\rho_{2}\right),
\end{gathered}
$$

and the coefficients $A_{1}-A_{8}$, being quite lengthy and not needed in the discussion of stability, have not been written here.

(a) For the potentially stable arrangement $\left(\rho_{2}<\rho_{1}\right)$, all the coefficients of (31) are positive. So, all the roots of (31) are either real and negative, or there are complex roots (which occur in pairs) with negative real parts and the rest negative real roots. The system is therefore stable in each case.

(b) For the potentially unstable arrangement $\left(\rho_{2}>\rho_{1}\right)$, the constant term in (31) is negative. Equation (31), therefore, allows at least one change of sign and so has a positive root and hence the system is unstable.

\section{EFFECT OF A VARIABLE HORIZONTAL MAGNETIC FIELD}

Consider the motion of incompressible, infinitely conducting Newtonian and Oldroydian viscoelastic fluids in the presence of suspended particles and a variable horizontal magnetic field $\vec{H}(H(z), 0,0)$. Let $\vec{h}\left(h_{x}, h_{y}, h_{z}\right)$ denote the perturbation in the magnetic field, then the linearized perturbation equations are

$$
\begin{aligned}
&\left(1+\lambda \frac{\partial}{\partial t}\right) \rho \frac{\partial \vec{v}}{\partial t} \\
&=\left(1+\lambda \frac{\partial}{\partial t}\right)[-\nabla \delta p+\vec{g} \delta \rho+K N(\vec{u}-\vec{v}) \\
&\left.+\frac{\mu_{e}}{4 \pi}\{(\nabla \times \vec{H}) \times \vec{h}+(\nabla \times \vec{h}) \times \vec{H}\}\right] \\
&+\mu\left(1+\lambda_{0} \frac{\partial}{\partial t}\right) \nabla^{2} \vec{v}, \\
& \quad \nabla \cdot \vec{h}=0, \\
& \frac{\partial \vec{h}}{\partial t}=\nabla \times(\vec{v} \times \vec{H}),
\end{aligned}
$$


together with (8)-(10). Assume that the perturbation $\vec{h}\left(h_{x}, h_{y}, h_{z}\right)$ in the magnetic field has also a space and time dependence of the form (12). $\mu_{e}$ stands for the magnetic permeability. Following the procedure as in Sections 3-5, we obtain

$$
A_{10} n^{10}+A_{9} n^{9}+\cdots+A_{1} n+A_{0}=0
$$

where

$$
\begin{aligned}
A_{10}= & \lambda \tau^{3}\left(\rho_{1}+\rho_{2}\right)^{2}, \\
A_{1}= & \left(1+\rho_{2}\right)\left[(\lambda+\tau)\left\{k_{x}^{2} V_{A}^{2}\left(\rho_{1}+\rho_{2}\right)-g k\left(\rho_{2}-\rho_{1}\right)\right\}\right] \\
& +\rho_{2} \tau\left[k_{x}^{2} V_{A}^{2}\left(\rho_{1}+\rho_{2}\right)-g k\left(\rho_{2}-\rho_{1}\right)\right] \\
& +\tau k_{x}^{2} V_{A}^{2}\left(\rho_{1}+\rho_{2}\right)+2 k^{2} v \rho_{1}\left[1+2 \tau\left(\rho_{1}+\rho_{2}\right)\right], \\
A_{0}= & \left(1+\rho_{2}\right)\left[k_{x}^{2} V_{A}^{2}\left(\rho_{1}+\rho_{2}\right)-g k\left(\rho_{2}-\rho_{1}\right)\right],
\end{aligned}
$$

and the coefficient $A_{2}-A_{9}$, being quite lengthy and not needed in the discussion of stability, have not been written here. Also, for the sake of simplicity, we have considered that the Alfvén velocities of the two fluids are the same so that

$$
V_{A}^{2}=\frac{\mu_{e} H_{1}^{2}}{4 \pi \rho_{1}}=\frac{\mu_{e} H_{2}^{2}}{4 \pi \rho_{2}} .
$$

\section{DISCUSSION}

(a) For the potentially stable arrangement $\left(\rho_{2}<\rho_{1}\right)$, (34) does not allow any positive root as there is no change of sign. The system is therefore stable. Thus when the ordinary (Newtonian) viscous fluid overlies Oldroydian viscoelastic fluid in the presence of suspended particles and a variable horizontal magnetic field, the system is stable for the potentially stable configuration.

(b) For the potentially unstable arrangement $\left(\rho_{2}>\rho_{1}\right)$, if

$$
k_{x}^{2} V_{A}^{2}\left(\rho_{1}+\rho_{2}\right)>g k\left(\rho_{2}-\rho_{1}\right),
$$

(34) does not admit any change of sign and so has no positive root. Therefore the system is stable. However, if

$$
k_{x}^{2} V_{A}^{2}\left(\rho_{1}+\rho_{2}\right)>g k\left(\rho_{2}-\rho_{1}\right),
$$

the constant term in (34) is negative. Equation (34), therefore, allows at least one change of sign and so has at least one positive root. The occurrence of a positive root implies that the system is unstable. Thus for the unstable case $\rho_{2}>$ $\rho_{1}$, the system is stable or unstable depending on whether $k_{x}^{2} V_{A}^{2}\left(\rho_{1}+\rho_{2}\right)$ is greater than or smaller than $g k\left(\rho_{2}-\rho_{1}\right)$. Thus, the magnetic field has got a stabilizing effect and completely stabilizes the wavenumber band $k>k^{*}$, where

$$
k^{*}=\frac{g\left(\rho_{2}-\rho_{1}\right)}{\left(\rho_{2}+\rho_{1}\right) V_{A}^{2}} \sec ^{2} \theta,
$$

and $\theta$ is the inclination of the wave vector $\vec{k}$ to the direction of the magnetic field $\vec{H}$, that is, $k_{x}=k \cos \theta$.

\section{ACKNOWLEDGMENT}

The authors are grateful to the referee for the critical comments which led to a significant improvement of the paper.

\section{REFERENCES}

[1] S. Chandrasekhar, Hydrodynamic and Hydromagnetic Stability, Dover, New York, NY, USA, 1981.

[2] P. K. Bhatia and J. M. Steiner, "Convective instability in a rotating viscoelastic fluid layer," Zeitschrift für Angewandte Mathematik und Mechanik, vol. 52, pp. 321-327, 1972.

[3] P. K. Bhatia and J. M. Steiner, "Thermal instability in a viscoelastic fluid layer in hydromagnetics," Journal of Mathematical Analysis and Applications, vol. 41, no. 2, pp. 271-283, 1973.

[4] R. C. Sharma, "Effect of rotation on thermal instability of a viscoelastic fluid," Acta Physiologica Hungarica, vol. 40, no. 1, pp. 11-17, 1976.

[5] J. W. Scanlon and L. A. Segel, "Some effects of suspended particles on the onset of Bénard convection," Physics of Fluids, vol. 16, no. 10, pp. 1573-1578, 1973.

[6] R. C. Sharma, K. Prakash, and S. N. Dube, "Effect of suspended particles on the onset of Benard convection in hydromagnetics," Acta Physiologica Hungarica, vol. 40, no. 1, pp. 310, 1976.

[7] V. I. Palaniswamy and C. M. Purushotham, "Stability of shear flow of stratified fluids with fine dust," Physics of Fluids, vol. 24, no. 7, pp. 1224-1228, 1981.

[8] H. Frohlish and R. Sack, Proceedings of the Royal Society of London. Series A, vol. 185, p. 415, 1946.

[9] J. G. Oldroyd, "The elastic and viscous properties of emulsions and suspensions," Proceedings of the Royal Society of London. Series A, vol. 218, no. 1132, pp. 122-132, 1953.

[10] J. G. Oldroyd, "Non-newtonian effects in steady motion of some idealized elastico-viscous liquids," Proceedings of the Royal Society of London. Series A, vol. 245, no. 1241, pp. 278297, 1958.

[11] B. A. Toms and D. J. Strawbridge, "Elastic and viscous properties of dilute solutions of polymethyl methacrylate in organic liquids," Transactions of the Faraday Society, vol. 49, no. 10, pp. 1225-1232, 1953.

[12] R. C. Sharma and K. C. Sharma, "Rayleigh-Taylor instability of two viscoelastic superposed fluids," Acta Physiologica Hungarica, vol. 45, no. 3, pp. 213-220, 1978.

[13] A. Kent, "Instability of laminar flow of a perfect magnetofluid," Physics of Fluids, vol. 9, no. 7, pp. 1286-1289, 1966.

[14] R. C. Sharma and P. Kumar, "Rayleigh-Taylor instability of viscous-viscoelastic fluids through porous medium," Indian Journal of Pure and Applied Mathematics, vol. 24, pp. 563-569, 1993.

[15] S. C. Agrawal and A. K. Goel, "Shear flow instability of viscoelastic fluid in a porous medium," Indian Journal of Pure and Applied Mathematics, vol. 29, pp. 969-981, 1998.

[16] P. K. Bhatia and R. P. Mathur, "Instability of viscoelastic superposed fluids in a vertical magnetic field through porous medium," Ganita Sandesh, vol. 17, pp. 21-32, 2003.

[17] P. Kumar, H. Mohan, and G. J. Singh, "Rayleigh-Taylor instability of rotating oldroydian viscoelastic fluids in porous medium in presence of a variable magnetic field," Transport in Porous Media, vol. 56, no. 2, pp. 199-208, 2004. 


\section{AUTHOR CONTACT INFORMATION}

Pardeep Kumar: Department of Mathematics, ICDEOL

Himachal Pradesh University, Summer-Hill, Shimla 171005, India;

drpardeep@sancharnet.in

Mahinder Singh: Department of Mathematics, ICDEOL

Himachal Pradesh University, Summer-Hill, Shimla 171005, India;

mahinder_singh91@rediffmail.com 